\title{
Selective Estrogen Receptor Modulators Enhance CNS Remyelination Independent of Estrogen Receptors
}

\author{
Kelsey A. Rankin, ${ }^{1}$ Feng Mei, ${ }^{1}$-Kicheol Kim, ${ }^{1}$ Yun-An A. Shen, ${ }^{1}$ Sonia R. Mayoral, ${ }^{1}$ CCaroline Desponts, ${ }^{2}$ \\ Daniel S. Lorrain, ${ }^{2}{ }^{\circledR}$ Ari J. Green, ${ }^{1}$ Sergio E. Baranzini, ${ }^{1}$ OJonah R. Chan, ${ }^{1}$ and Riley Bove ${ }^{1}$ \\ ${ }^{1}$ Weill Institute for Neurosciences, Department of Neurology, University of California, San Francisco, California 94143, and ${ }^{2}$ Inception Sciences, San Diego, \\ California 92121
}

A significant unmet need for patients with multiple sclerosis (MS) is the lack of U.S. Food and Drug Administration (FDA)-approved remyelinating therapies. We have identified a compelling remyelinating agent, bazedoxifene (BZA), a European Medicines Agency (EMA)-approved (and FDA-approved in combination with conjugated estrogens) selective estrogen receptor (ER) modulator (SERM) that could move quickly from bench to bedside. This therapy stands out as a tolerable alternative to previously identified remyelinating agents and other candidates within this family. Using an unbiased high-throughput screen, with subsequent validation in both murine and human oligodendrocyte precursor cells (OPCs) and coculture systems, we find that BZA enhances differentiation of OPCs into functional oligodendrocytes. Using an in vivo murine model of focal demyelination, we find that BZA enhances OPC differentiation and remyelination. Of critical importance, we find that BZA acts independently of its presumed target, the ER, in both in vitro and in vivo systems. Using a massive computational data integration approach, we independently identify six possible candidate targets through which SERMs may mediate their effect on remyelination. Of particular interest, we identify EBP (encoding $3 \beta$-hydroxysteroid- $\Delta 8, \Delta 7$ isomerase), a key enzyme in the cholesterol biosynthesis pathway, which was previously implicated as a target for remyelination. These findings provide valuable insights into the implications for SERMs in remyelination for MS and hormonal research at large.

Key words: multiple sclerosis; myelin; oligodendrocytes

\section{Significance Statement}

Therapeutics targeted at remyelination failure, which results in axonal degeneration and ultimately disease progression, represent a large unmet need in the multiple sclerosis (MS) population. Here, we have validated a tolerable European Medicines Agency-approved (U.S. Food and Drug Administration-approved in combination with conjugated estrogens) selective estrogen receptor (ER) modulator (SERM), bazedoxifene (BZA), as a potent agent of oligodendrocyte precursor cell (OPC) differentiation and remyelination. SERMs, which were developed as nuclear ER- $\alpha$ and ER- $\beta$ agonists/antagonists, have previously been implicated in remyelination and neuroprotection, following a heavy focus on estrogens with underwhelming and conflicting results. We show that nuclear ERs are not required for SERMs to mediate their potent effects on OPC differentiation and remyelination in vivo and highlight EBP, an enzyme in the cholesterol biosynthesis pathway that could potentially act as a target for SERMs.

\section{Introduction}

In multiple sclerosis (MS), an inflammatory autoimmune disorder of the CNS, an adaptive immune response to unknown CNS

Received June 11, 2018; revised Jan. 17, 2019; accepted Jan. 20, 2019.

Author contributions: K.A.R. wrote the first draft of the paper; K.A.R., J.R.C., and R.B. edited the paper; K.A.R., Y.-A.A.S., A.J.G., J.R.C., and R.B. designed research; K.A.R., F.M., K.K., Y.-A.A.S., S.R.M., C.D., D.S.L., and S.E.B. performed research; F.M. and J.R.C. contributed unpublished reagents/analytic tools; K.A.R. analyzed data; K.A.R. wrote the paper.

This work was supported by the National Institutes of Health/National Institute of Neurological Disorders and Stroke (Grants R01NS062796, R01NS097428, R01NS095889, and R01NS088155), the National Multiple Sclerosis Society (Grant RG5203A4), the Adelson Medical Research Foundation (ANDP Grant A130141), the Heidrich Family and Friends, and the Rachleff Family Endowments. We thank the Innovation Program for Remyelination and Repair at the Weill Institute for Neurosciences at the University of California-San Francisco.

The authors declare no competing financial interests. antigens results in oligodendrocyte damage, loss of myelin, and neurologic dysfunction (Hauser et al., 2013). Because it has been shown that existing oligodendrocytes are unable to participate in repair (Crawford et al., 2016), newly differentiated oligodendrocytes are required. In the brains of MS patients, oligodendrocyte precursor cell $(\mathrm{OPC})$ proliferation and migration to the lesion site go unhindered, but OPCs are subsequently unable to differentiate into functional oligodendrocytes (Kuhlmann et al., 2008; Frischer et al., 2015). It is generally accepted that disability pro-

Correspondence should be addressed to Jonah R. Chan at Jonah.Chan@ucsf.edu or Riley Bove at Riley.Bove@ucsf.edu.

https://doi.org/10.1523/JNEUROSCl.1530-18.2019

Copyright $\odot 2019$ the authors $\quad 0270-6474 / 19 / 392184-11 \$ 15.00 / 0$ 
gression is associated with axon degeneration resulting from remyelination failure (Irvine and Blakemore, 2008; Trapp and Nave, 2008). There are currently no remyelinating therapeutics approved for patients: a clear unmet need in a disease afflicting 850,000 people in the United States and 2.3 million worldwide (Wallin, 2017). To target the OPC differentiation block and promote remyelination, we sought to repurpose current U.S. Food and Drug Administration (FDA)-approved compounds.

\section{Materials and Methods Micropillar arrays and quantification of differentiation-promoting compounds}

Micropillar arrays were fabricated via previously reported methodology (Mei et al., 2014) into 96- or 384-well plates. To test compound effects on OPC proliferation or differentiation, microarrays were coated with polyL-lysine (Sigma-Aldrich) for $1 \mathrm{~h}$, then washed 3 times with water. In each well, 40,000 or 150,000 rat OPCs were seeded, respectively. These OPCs were maintained in medium containing DMEM (Invitrogen), B27 (Invitrogen), N2 (Invitrogen), $\mathrm{N}$-acetylcysteine (Sigma-Aldrich), forskolin (Sigma-Aldrich), and penicillin-streptomycin (Invitrogen). Medium was supplemented with PDGF-AA (PeproTech) overnight before removal and treatment with candidate FDA-approved small molecules. For the preliminary unbiased screen, compounds were diluted to $1 \mu \mathrm{M}$ and left in medium for $3 \mathrm{~d}$ before immunostaining. Each compound was tested in triplicate. For the curated, biased screen of SERMs, candidate compounds were diluted to $500 \mathrm{~nm}$ and left in medium for $2 \mathrm{~d}$ before immunostaining. Each compound was repeated in duplicate. Myelin basic protein (MBP) or PDGFR $\alpha$ staining was visualized via $20 \times$ microscopy using Zeiss Axio Imager Z1 microscope.

\section{Purification and culturing of oligodendrocyte precursor cells} Oligodendrocyte precursor cell (OPC) purification was conducted per a previously validated protocol (Mei et al., 2014). Briefly, OPCs were isolated from the cortical hemispheres of postnatal day 7 rat or mouse brains. Cortical tissue was mechanically dissected and immersed in papain (Worthington) at $37^{\circ} \mathrm{C}$ for $75 \mathrm{~min}$ with periodic shaking. Suspension was then triturated and immersed in $0.2 \%$ BSA (Sigma-Aldrich) to undergo sequential immunopanning consisting of negative selection for $30 \mathrm{~min}$ and positive selection for $45 \mathrm{~min}$ at room temperature. Tissue culture plates for selection were incubated with goat IgG and IgM secondary antibodies (Jackson ImmunoResearch) in $50 \mathrm{~mm}$ Tris-HCl, pH 9.5, at room temperature overnight, washed with Dulbecco's PhosphateBuffered Saline (Invitrogen) 3 times, and then incubated at room temperature in primary antibodies Ran-2 and Gal-C (negative selection) or O4 (positive selection). Resultant OPCs were dissociated from the positive selection dish via $0.05 \%$ trypsin (Invitrogen). Reaction was halted using 30\% FBS (Invitrogen). OPCs using this methodology are $>95 \%$ pure. Purified OPCs were seeded onto poly-L-lysine-coated (SigmaAldrich) $25 \mathrm{~mm}^{2}$ coverslips at a density of 200,000 cells/coverslip in medium supplemented with DMEM (Invitrogen), B27 (Invitrogen), N2 (Invitrogen), $\mathrm{N}$-acetylcysteine (Sigma-Aldrich), forskolin (Sigma-Aldrich), penicillin-streptomycin (Invitrogen), and PDGF-AA (Peprotech) overnight at $37^{\circ} \mathrm{C}$ and $5 \% \mathrm{CO}_{2}$. OPCs were then treated with compound or control following PDGF-AA (Peprotech) removal for $2 \mathrm{~d}$. Vehicle control preparation included the dilution of vehicle (dimethylsulfoxide, DMSO) into culture medium to equivalent concentrations as treatment groups.

\section{Purification of dorsal root ganglion neurons, coculture system}

Dorsal root ganglia (DRGs) were isolated following a previously validated methodology (Chan et al., 2004). Briefly, DRGs were isolated from the spinal column of embryonic day 15 rat embryos via dissection. Isolated DRGs were immersed in $0.25 \%$ trypsin (Invitrogen) at $37^{\circ} \mathrm{C}$ for 45 min, resuspended in L15 + FBS (Invitrogen), dissociated, and plated onto collagen-coated coverslips at a density of 150,000 cells $/ 25 \mathrm{~mm}^{2}$ coverslip in M1 medium containing MEM (Invitrogen), glucose (SigmaAldrich), and Hyclone FBS (Fisher Scientific) plus neural growth factor
(NGF) (AbD Serotec). DRGs were allowed to proliferate and maintained in culture for 3 weeks to become NGF independent. Medium was alternated every $3 \mathrm{~d}$ between M1 medium and E2F medium containing MEM (Invitrogen), glucose (Sigma-Aldrich), 5-fluorodeoxyuridine (SigmaAldrich), and NGF (AbD Serotec) to select for nondividing cells only. Once DRGs are NGF independent, they are ready for coculture. For coculture systems, the medium was switched to OPC medium containing DMEM (Invitrogen), B27 (Invitrogen), N2 (Invitrogen), $\mathrm{N}$-acetylcysteine (Sigma-Aldrich), forskolin (Sigma-Aldrich), and penicillin-streptomycin (Invitrogen) without PDGF-AA and was supplemented every $3 \mathrm{~d}$. OPCs were allowed to proliferate until they reached optimal confluence around $7 \mathrm{~d}$, whereupon they were treated with either $\mathrm{BZA}$ (Invitrogen) or control for $5 \mathrm{~d}$.

\section{Immunostaining, image acquisition, and fluorescence quantifications}

For immunostaining, cell cultures were first fixed in 4\% PFA (EM Science), dehydrated, and then permeabilized using a blocking solution containing 20\% goat serum (Sigma-Aldrich) and $0.2 \%$ Triton X-100 (Sigma-Aldrich) for $2 \mathrm{~h}$. Cultures were then washed and placed in primary antibody solution in $20 \%$ goat serum (Sigma-Aldrich) for $2 \mathrm{~h}$. Oligodendrocytes were identified using the primary rat monoclonal antibody to MBP (1:200; EMD Millipore) or the mouse monoclonal antibody to CC1 (1:500; EMD Millipore). OPCs were identified via the primary rabbit monoclonal antibody against PDGFR $\alpha$ (1:8000; gift from W.B. Stallcup). Neurons were detected via the primary mouse monoclonal antibody against neurofilament (NF; 1:500; Covance). The paranodal region for internode quantification was detected via the primary rabbit polyclonal antibody against Contactin associated protein 1 (Caspr; 1:800; Abcam). Alexa Fluor 488, Alexa Fluor 594, and Alexa Fluor 647 IgG secondary antibodies against rat, rabbit, and mouse (1:1000; Invitrogen) were used for fluorescence detection. Cell bodies were determined via DAPI (Vector Laboratories) administered for 15 min during secondary antibody incubation in $20 \%$ goat serum (Sigma-Aldrich) for $1 \mathrm{~h}$. Images were obtained using a Zeiss Axio Imager Z1 microscope with the appropriate excitation wavelengths for the secondary antibodies $(488,568,628$, and $380 \mathrm{~nm}$ ). Quantifications were determined via randomly selected fields of view under $20 \times$ magnification.

For lysolecithin, the in vivo demyelination model, remyelination was first assessed via immunostaining. Floating coronal brain sections (30 $\mu \mathrm{m}$ ) were permeabilized using a solution of $20 \%$ goat serum (SigmaAldrich) and $0.2 \%$ Triton X-100 (Sigma-Aldrich) for $2 \mathrm{~h}$ at room temperature. Floating sections were then placed in a solution containing $0.2 \%$ Triton X-100 (Sigma-Aldrich) and primary antibodies in $20 \%$ goat serum (Sigma-Aldrich) overnight on a shaker at $4^{\circ} \mathrm{C}$. Oligodendrocytes were determined based on the primary mouse monoclonal antibody against CC1 (1:500; EMD Millipore). Myelin was determined via the primary human monoclonal antibody against myelin oligodendrocyte glycoprotein (MOG; 1:500; EMD Millipore). Axon presence was verified using the primary rabbit polyclonal antibody against NF (1:1000; Covance). The Alexa Fluor 488, Alexa Fluor 594, and Alexa Fluor 647 IgG secondary antibodies against rat, rabbit, human, and mouse (1:1000; Invitrogen) were used for fluorescence detection. Cell bodies were determined via DAPI (Vector Laboratories) administered for 15 min during secondary antibody incubation in $20 \%$ goat serum (Sigma-Aldrich) for $1 \mathrm{~h}$. Lesioned areas were determined via DAPI (Vector Laboratories) for cell bodies of infiltrating cells.

\section{Lysolecithin in vivo focal demyelination model in the corpus callosum}

Focal demyelinated lesions were induced using a previously validated methodology (Fancy et al., 2011). Briefly, 8-week-old female mice were anesthetized using isoflurane (Henry Schein) and buprenorphine (Henry Schein). Using stereotaxic equipment, chemical demyelination was induced in the white matter tract of the corpus callosum at the following coordinates: AP: $-1.04 \mathrm{~mm}, \mathrm{ML}: 1 \mathrm{~mm}$, and DV: $-1.75 \mathrm{~mm}$ from bregma using a Hamilton syringe in female 8-week-old C57BL।6 WT and $\mathrm{ER} \alpha$ and $\beta$ double-knock-out (DKO) female mice using $1 \mu \mathrm{l}$ of 
$1 \%$ lysolecithin (L- $\alpha$-lysophatidylcholine; Sigma-Aldrich) solution. Following the demyelinating event, mice were treated with either vehicle or $\mathrm{BZA}$ (Invitrogen) at a dose of $10 \mathrm{mg} / \mathrm{kg} / \mathrm{d}$ for $7 \mathrm{~d}$ via oral gavage (DMSO $<10 \%$ of solution; solution in water), based on conversion from human equivalent dose (40 $\mathrm{mg}$ daily) to animal equivalent dose $(7.24 \mathrm{mg} / \mathrm{kg}$ ) (Nair and Jacob, 2016). Because clinical trials were conducted for 2 years (Pickar et al., 2009) and we hoped to see an observable difference in animals after $7 \mathrm{~d}$ of treatment, we increased this value to $10 \mathrm{mg} / \mathrm{kg} / \mathrm{d}$ using a comparable treatment paradigm to the previously published clemastine remyelination procedure (Mei et al., 2016). Treatment began at $1 \mathrm{~d}$ postlesion $(\mathrm{dpl})$ and ended at $7 \mathrm{dpl} .5 \mathrm{WT}$ animals were treated with BZA (Invitrogen) and 5 WT with vehicle (DMSO in water). For ER-DKO experiments, $n=4$ for DKO animals, and $n=6$ for WT. Ten images were analyzed for each group.

Animals were killed and perfused at $10 \mathrm{dpl}$. For this, they were anesthetized and flushed with $1 \times$ PBS and then perfused transcardially using $4 \%$ paraformaldehyde (PFA) (Electron Microscopy Sciences) until stiff. Brains were dissected out of the animals and postfixed in 4\% PFA (Electron Microscopy Sciences) at $4^{\circ} \mathrm{C}$ overnight. Brains were then switched to a solution of $30 \%$ sucrose (Sigma-Aldrich). Once sucrose had adequately seeped into the tissue, brain sections of the relevant area were obtained. Coronal sections for floating immunostaining were cut at $30 \mu \mathrm{m}$ intervals using a microtome-sectioning tool, with brains immersed in a solution of 2:1 30\% sucrose: optimal cutting temperature solution (Sigma-Aldrich). Relevant sections were determined using the mouse brain atlas and scanning under a microscope for evidence of demyelination.

\section{Electron microscopy (EM)}

Mice were perfused transcardially with $1.25 \%$ glutaraldehyde (Electron Microscopy Sciences), 2\% PFA (Electron Microscopy Sciences) in $0.1 \mathrm{M}$ sodium cacodylate (Ladd Research) at pH 7.4. Mice were preflushed with $0.1 \mathrm{~m}$ sodium cacodylate (Ladd Research). Tissue was sent for processing to the San Francisco Veterans Affairs Medical Center. Coronal sections of corpus callosum were postfixed in $2 \%$ aqueous osmium tetroxide and stained en bloc with $2 \%$ aqueous uranyl acetate. Sections were then dehydrated in ethanol, cleared in propylene oxide, and embedded in Eponate (Ted Pella). To identify lesion areas, semithin sections were stained with toluidine blue. Once lesion areas were identified via light microscope, ultrathin sections were obtained using a Leica Ultracut E microtome. These ultrathin sections were then stained with uranyl acetate and Reynold's lead and mounted onto grids. Grids were examined using a Philips Tecnai 10 electron microscope. Lesion demarcation was determined based on myelinated axon density and the appearance of unmyelinated and thinly myelinated axons. Lesion borders were evident based on a line of normal distribution of myelinated axons juxtaposed with the absence of myelinated axons, a clear focal lesion, and stark visual difference. This was evident in all cases. Remyelinated axons were identified within this focal lesion. Many remyelinated axons within the lesions were above the canonical g-ratio threshold of 0.8 , but some were also slightly below this number. We chose to include all myelinated axons within the obvious boundaries of the identified focal lesions because, presumably, all axons within the focal lesion are demyelinated. g-ratios were determined using the ratio of the Feret diameter of axons to the Feret diameter of axons with the myelin sheath. Feret diameter was calculated by ImageJ software as "the longest distance between any two points along the selection boundary" (https://imagej.nih.gov/ij/docs/ menus/analyze.html). Analyses took 6 areas for each group of 2 pairs of mice that had undergone lysolecithin administration, followed by treatment with either BZA $(10 \mathrm{mg} / \mathrm{kg} / \mathrm{d})$ or vehicle from 1 to $7 \mathrm{dpl}$. Mice were perfused at $10 \mathrm{dpl}$.

\section{KO mice}

All rodents in this study were handled following the approval of the University of California San Francisco Administrative Panel on Laboratory Animal Care. All mice used in the study were of the C57BL $\backslash 6$ background. $\mathrm{ER} \alpha$ and $\mathrm{ER} \beta$ mice were obtained as a generous gift from the Shah laboratory (Nirao Shah, Stanford University) and are additionally available via The Jackson Laboratory (https://www.jax.org/strain/
004744; https://www.jax.org/strain/004745). Briefly, global ESR1 KO $(\mathrm{ER} \alpha)$ mice were generated via an insertional disruption mutation in the Esrl gene, which codes for the nuclear estradiol hormone receptor $\alpha$, where exon 2 is deleted and several stop codons are inserted (The Jackson Laboratory). Both female and male animals homozygous for the ER $\alpha$ mutant allele are infertile. Global KO animals have been previously validated by The Jackson Laboratory by determining 4 -fold higher serum estradiol levels (indicative of estrogen insensitivity), only 3\% estrogen binding in uterine tissue, and no observable estrogen binding in brain, kidney, or liver in homozygous KOs (The Jackson Laboratory). Global ESR2 KO $(E R \beta)$ mice were generated via the insertion of stop codons into exon 3 of the Esr2 gene, which codes for the nuclear estradiol hormone receptor $\beta$ (The Jackson Laboratory). Females homozygous for the ER $\beta$ mutant allele are subfertile, whereas males are normal. Global KO animals have been systematically validated by The Jackson Laboratory via the absence of ESR2 protein (The Jackson Laboratory). Briefly, KOs were determined via the presence of WT or mutant allele via PCR amplification and visualization on $2 \%$ agarose gel (Invitrogen). ER $\alpha$ mutant allele was $300 \mathrm{bp}$ and the WT allele was $234 \mathrm{bp}$ in length, as determined by the following PCR probes: common: 5' -TAC GGC CAG TCG GGC ATC-3'; WT: 5' -GTA GAA GGC GGG AGG GCC GGT GTC-3'; mutant: 5' -GCT ACT TCC ATT TGT CAC GTC C- $3^{\prime}$. The ER $\beta$ mutant allele was 160 bp in length and the WT allele was $100 \mathrm{bp}$, as determined by the following PCR probes: common: 5' -TCA CAG GAC CAG ACA CCG TA-3'; WT: 5'-GTT GTG CCA GCC CTG TTA CT-3'; mutant: 5'-GCA GCC TCT GTT CCA CAT ACA C-3'.

\section{Human OPC development from ES cells}

Human OPCs were derived from the embryonic stem cell (ESC) H1 line following a previously validated methodology (Douvaras and Fossati, 2015). Briefly, ESCs were cultured on 6-well Matrigel (Fisher Scientific)coated plates in mTeSR medium (STEMCELL Technologies) for $2 \mathrm{~d}$. Medium was then supplemented with SB431542 (SelleckChem), LDN189193 (SelleckChem), and retinoic acid (Sigma-Aldrich) for $7 \mathrm{~d}$. Medium was then changed to DMEM/F12 (Life Technologies), N2 (Invitrogen), retinoic acid (Sigma-Aldrich), and smoothened agonist (EMD Millipore) for $4 \mathrm{~d}$. Cells were then removed from adherent culture, and incubated in nonadherent dishes to generate embryoid bodies (EBs) in medium containing DMEM/F12 (Life Technologies) supplemented with N2 (Invitrogen), B27 (Invitrogen), retinoic acid (Sigma-Aldrich), and smoothened agonist (EMD Millipore). After $8 \mathrm{~d}$, medium was changed to medium containing DMEM/F12 (Life Technologies), N2 (Invitrogen), B27 (Invitrogen), PDGF-AA (R\&D Systems), IGF-1 (R\&D Systems), HGF (EMD Millipore), NT3 (EMD Millipore), T3 (SigmaAldrich), biotin (Sigma-Aldrich), cAMP (Sigma-Aldrich), and insulin (Sigma-Aldrich). Ten days later, EBs were plated onto poly-L-ornithine (Sigma-Aldrich) and laminin (Thermo Fisher Scientific)-coated 24-well plates at a ratio of $2 \mathrm{EBs} / \mathrm{cm}^{2} .29 \mathrm{~d}$ later, medium was changed to DMEM/ F12 (Life Technologies), N2 (Invitrogen), B27 (Invitrogen), HEPES (Life Technologies), biotin (Sigma-Aldrich), cAMP (Sigma-Aldrich), insulin (Sigma-Aldrich), and ascorbic acid (Sigma-Aldrich) for $21 \mathrm{~d}$. FACSpurified $\mathrm{O}^{+}{ }^{+}$hOPCs were seeded onto poly-L-lysine (Sigma-Aldrich)coated coverslips and treated with $500 \mathrm{~nm}$ BZA (Invitrogen) for $5 \mathrm{~d}$.

\section{Bioinformatics profiling of SERMs and estrogens}

Data on how select remyelinating and nonremyelinating compounds bind to or regulate their molecular targets was extracted from HetioNet, a heterogeneous network containing 47,031 biomedical concepts (nodes) and 2,250,197 relationships (edges) among them curated from 29 publicly available databases (Himmelstein et al., 2017). In addition, the diseases related to the genes/proteins (Disease-associates-Gene_DaG) targeted by these compounds were also extracted. Specifically, Compound-binds-Gene (CbG), Compound-downregulates-Gene (CdG), and Compound-UpregulatesGene $(\mathrm{CuG})$ edges were queried for select remyelinating and nonremyelinating compounds from HetioNet. CbG edges were aggregated from BindingDB (Chen et al., 2001; Gilson et al., 2016), DrugBank (Law et al., 2014), and DrugCentral (Ursu et al., 2017). Only binding relationships to single proteins with affinities of at least $1 \mathrm{~mm}$ (as determined by $K_{\mathrm{d}}, K_{\mathrm{i}}$, or 


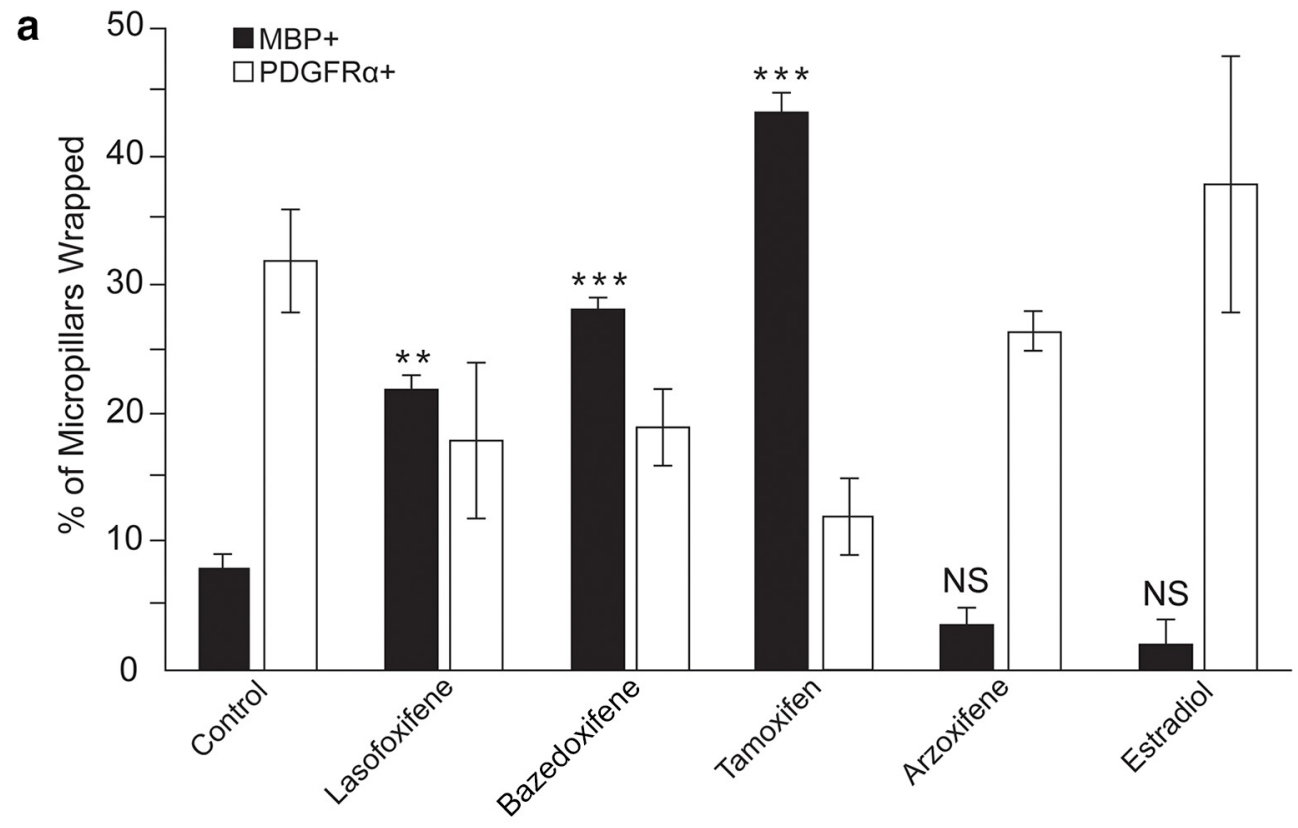

\begin{tabular}{|c|c|c|c|c|}
\hline Name & Categorization & Primary ER Type & FDA Approval & OPC Differentiation \\
\hline Bazedoxifene & SERM & Agonist/Antagonist & Yes, with CE & Yes \\
\hline Raloxifene & SERM & $\begin{array}{l}\text { ERa Agonist/Antagonist; } \\
\text { ERß Antagonist }\end{array}$ & Yes & Yes \\
\hline Tamoxifen & SERM & Agonist/Antagonist & Yes & Yes \\
\hline Toremifene & SERM & Agonist/Antagonist & Yes & Yes \\
\hline Clomiphene & SERM & Agonist/Antagonist & Yes & Yes \\
\hline Lasofoxifene & SERM & Agonist/Antagonist & No & Yes \\
\hline Ospemifene & SERM & Agonist/Antagonist & Yes & No \\
\hline$D P N$ & SERM & $E R \beta$ Agonist & No & No \\
\hline Arzoxifene & SERM & Agonist/Antagonist & No & No \\
\hline Fulvestrant & $\begin{array}{l}\text { Selective Estrogen } \\
\text { Receptor Degrader }\end{array}$ & Degrader & Yes & No \\
\hline Ethinyl Estradiol & Estrogen & Agonist & Yes & No \\
\hline Estrone & Estrogen & Agonist & Yes & No \\
\hline Estradiol & Estrogen & Agonist & Yes & No \\
\hline Mestranol & Estrogen & Agonist & Yes & No \\
\hline Erteberel & Estrogen & ERß Agonist & No & No \\
\hline Estriol & Estrogen & Agonist & No & No \\
\hline
\end{tabular}

Figure 1. Several candidate SERMs promote OPC differentiation and have translational potential. $\boldsymbol{a}$, Graph representing the percentage of micropillars wrapped following the repetition of the original binary indicant for myelination using micropillar arrays (BIMA) screen using a novel, curated list of candidate SERMs. This graph represents the quantification of the number of micropillars wrapped by either OPCS (PDGFR $\alpha$ ) or oligodendrocytes (MBP). $\boldsymbol{b}$, Summary of all SERMs tested, their previously reported mechanism of action, their FDA approval status, and their effects on OPC differentiation. Error bars indicate SEM. ${ }^{* *} p<0.01,{ }^{* *} p<0.001$. For all experiments, $n=2$ temporally separate trials; $n=2$ microarrays per group per trial.

$\mathrm{IC}_{50}$ ) were selected from the October 2015 release of BindingDB. Target, carrier, transporter, and enzyme interactions with single proteins (i.e., excluding protein groups) were extracted from DrugBank 4.2. In addition, all mapping DrugCentral target relationships were included. DaG edges were extracted from the GWAS Catalog (MacArthur et al., 2017), DISEASES (Pletscher-Frankild et al., 2015), DisGeNET (Piñero et al., 2017), and DOAF (Himmelstein, 2016) databases. CuG and CdG edges were extracted from the LINCS1000 Project (National Institutes of Health, 2018), which profiled the transcriptional response to thousands of small-molecule and genetic interference perturbations.

\section{Statistical analysis, blinding, and randomization}

All relevant statistical analyses were performed in Excel or Prism 7. The primary statistical test used was two-tailed Student's $t$ test. Significance is shown in the figures as: ${ }^{\star} p<0.05,{ }^{* *} p<0.01$, and ${ }^{* *} p<0.001$. In relevant analyses (specifically the results as reported in Figs. $1 a, 2 d, e, 4 c$, $5 c, d$, and $6 d$ ), adjustments were made for multiple comparisons using a one-way ANOVA followed by Tukey's multiple-comparisons test. All $p$-values were reported directly within the text. Treatment groups were compared with vehicle control groups. All experiments had $n \geq 2$. For quantifications, all investigators were appropriately blinded before the image acquisition phase and blinding was only reversed upon completion of all relevant analyses. For in vitro analyses, coverslips were randomly assigned to treatment groups and were excluded if deemed different in any way from selected coverslips (cell density, cell health, contamination). Animals used were assigned to treatment groups randomly and were excluded if perfusion was deemed inadequate.

\section{Study approval}

All rodents in this study were handled following the approval of the University of California San Francisco Administrative Panel on Laboratory Animal Care.

\section{Results}

An earlier high-throughput screen of $>1000$ FDA-approved bioactive molecules revealed not only anti-muscarinic compounds (Mei et al., 2014), but a previously unreported second cluster that 
a

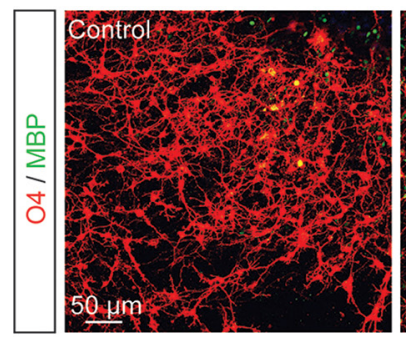

C
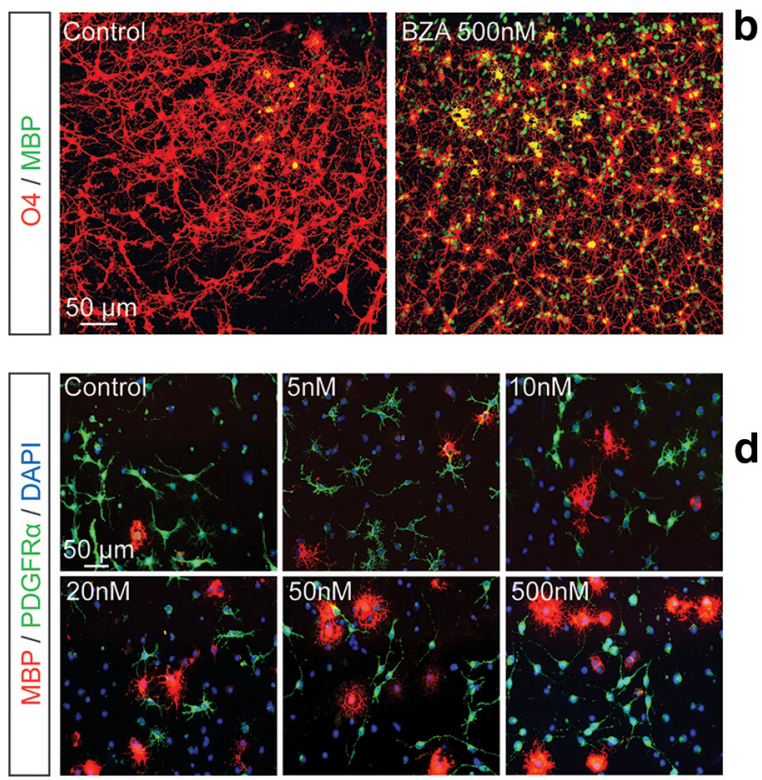

b

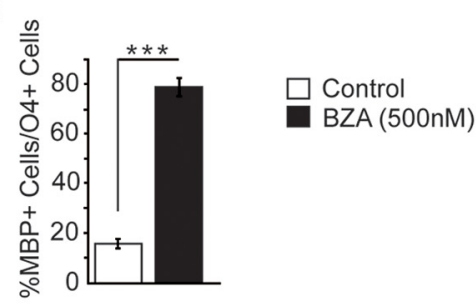

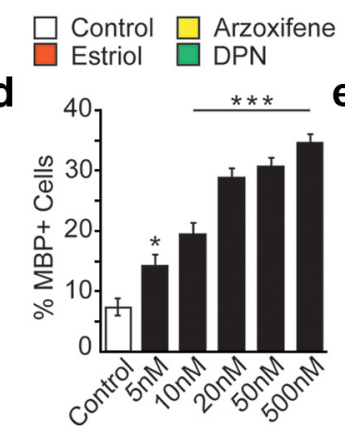
$\square$ Lasofoxifene Tamoxifen

BZA

e
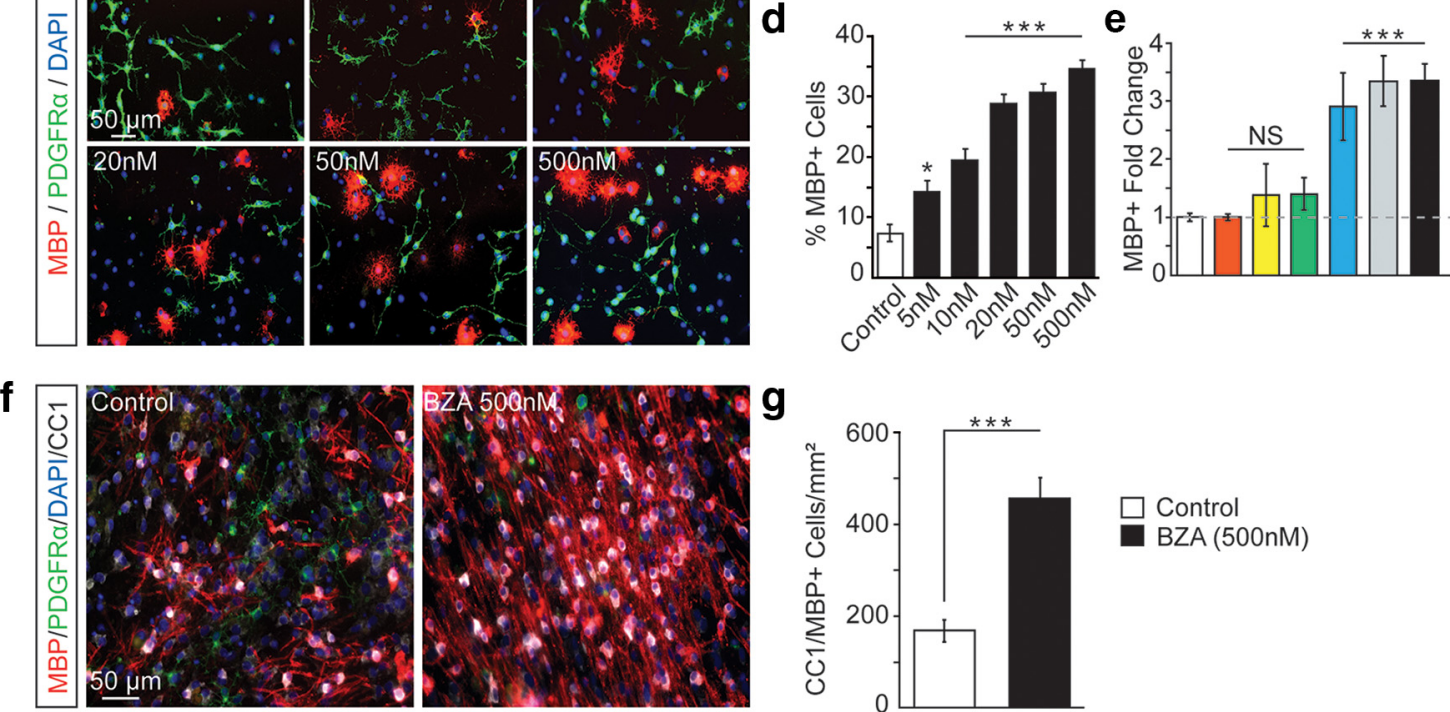

9

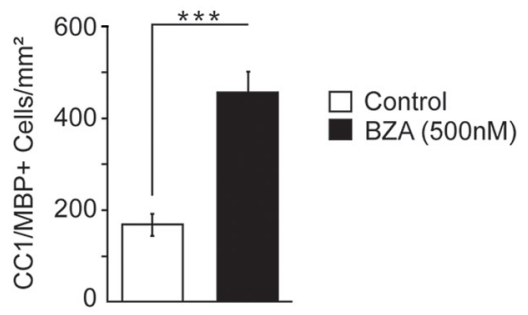

h
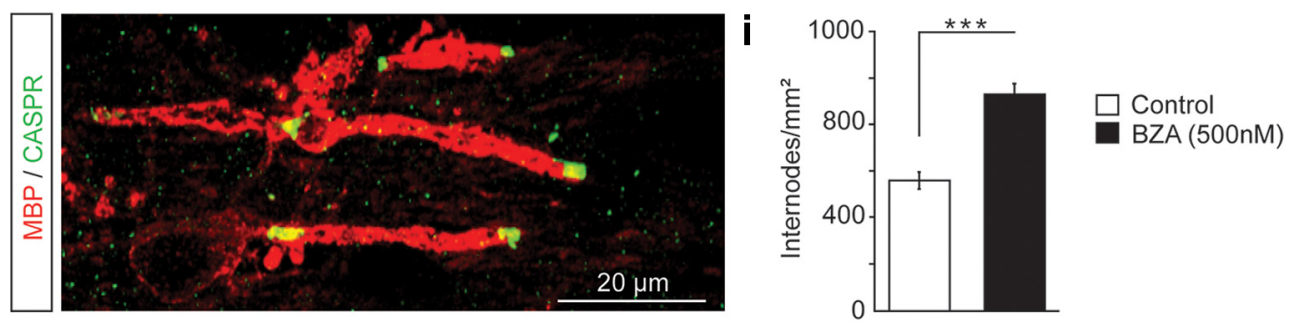

Figure 2. BZA enhances OPC differentiation in vitro. $a$, Representative images of control and BZA-treated ( $500 \mathrm{~nm}$ ) cultures of human OPCs treated for $5 \mathrm{~d}$ before immunostaining for 04 (red) and $\mathrm{MBP}$ (green). $\boldsymbol{b}$, Quantification of $\mathrm{MBP}^{+}$cells over total $04^{+}$cells. c, Representative images of rat OPC cultures and cultures treated with BZA at 5, 10, 20, 50, or 500 nM or vehicle control for $2 \mathrm{~d}$ and immunostained for MBP (red) and PDGFR $\alpha$ (green; an OPC marker). Cell nuclei are shown by DAPI (blue). $d$, Quantification of MBP ${ }^{+}$cells over total cells (DAPI ${ }^{+}$nuclei) for control cultures and 5-500 nм BZA. e, Quantification of fold-change of $\mathrm{MBP}^{+}$cells over total cells (DAPI ${ }^{+}$nuclei) for control cultures and additional candidate SERMs as well as estriol at $500 \mathrm{~nm} . \boldsymbol{f}$, Representative images of control and BZA-treated ( $500 \mathrm{~nm}$ ) OPC/DRG cocultures immunostained for MBP (red), CC1 (white), and PDGFR $\alpha$ (green). Cell nuclei are shown by DAPI (blue). $\boldsymbol{g}$, Quantification of CC1 and MBP colabeled cells per square millimeter. $\boldsymbol{h}$, Representative images displaying CASPR staining clustered in the paranodal region of myelin sheaths in BZA-treated cocultures. $\boldsymbol{i}$, Quantification of internodes per square millimeter in vehicle- and BZA-treated groups. Error bars indicate SEM. ${ }^{*} p<0.05,{ }^{* * *} p<0.001$. For all experiments, $n=2$ temporally separate trials; $n>3$ coverslips per group per trial.

significantly enhanced differentiation of rat OPCs: SERMs. SERMs have previously been implicated in remyelination and neuroprotection (Crawford et al., 2010; Khalaj et al., 2013; Moore et al., 2014; Gonzalez et al., 2016). Because we sought to bring treatment to patients at an accelerated timeline, we repeated a selective screen of FDA-approved members of this family, including some that had yet to be explored (Fig. 1). These results indicated that certain SERMs promoted OPC differentiation, whereas others and estradiol did not (lasofoxifene: $p=0.003$; bazedoxifene: $p=3.34 \times 10^{-4}$; tamoxifen: $p=7.27 \times 10^{-6}$; arzoxifene: $p=0.422$; estradiol: $p=0.189$, one-way ANOVA with Tukey's multiple-comparisons test). These results led us to concentrate on one candidate SERM: BZA, a nonsteroidal, thirdgeneration European Medicines Agency (EMA)-approved SERM (Wyeth Europa, Ltd., Conbriza [package insert], 2009), which in combination with conjugated estrogens is FDA approved in menopausal women (Wyeth Pharmaceuticals, Inc., and Pfizer, Inc.: Duavee [package insert], 2013; Mirkin and Komm, 2013). Compared with other high-yield SERMs, BZA is exceptionally well tolerated in clinical trials (McKeand et al., 2014) and thus represents an exciting therapy for which the path from "bench to bedside" could be streamlined. Of note, tamoxifen exhibits strong menopause-like side effects when given to women with estrogen-responsive breast cancers and the risk:benefit ratio has previously limited its treatment duration to 5 years (Perez, 2007).

To validate BZA's cell-autonomous effects on OPCs and to ensure its translational application, human embryonic stem cell (ESC) derived-OPCs (Douvaras and Fossati, 2015) were treated with BZA for $5 \mathrm{~d}$ (Fig. 2a). We saw significantly enhanced OPC differentiation into mature MBP (a marker of mature oligoden- 
a

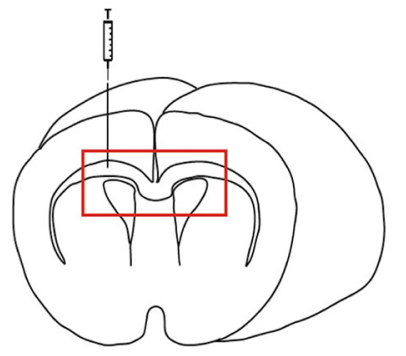

C $\square$ Control $\square \mathrm{BZA}(10 \mathrm{mg} / \mathrm{kg} /$ day $)$

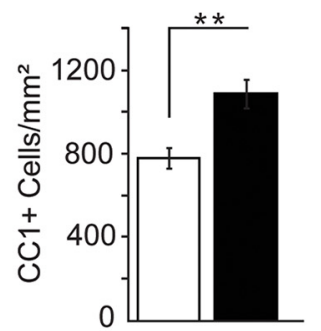

d

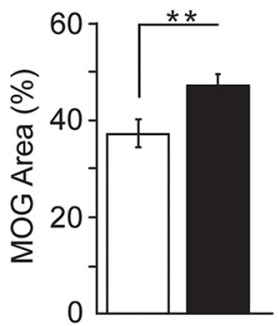

b

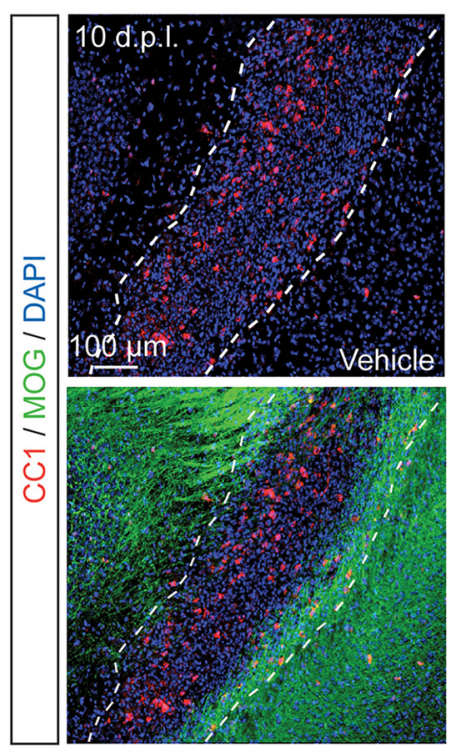

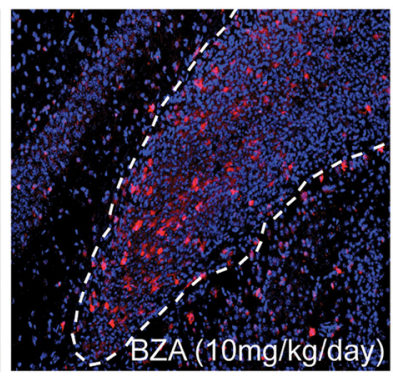

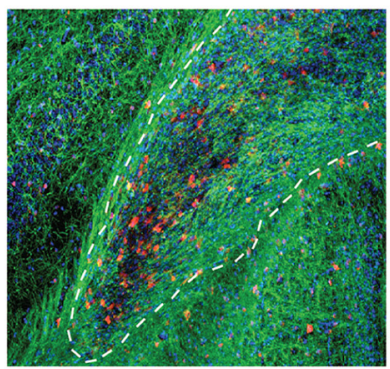

e

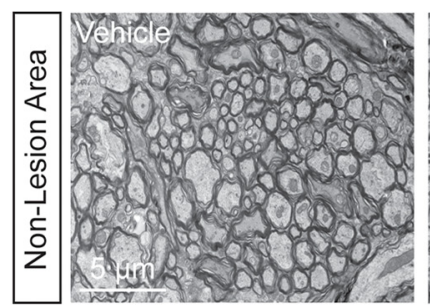

i

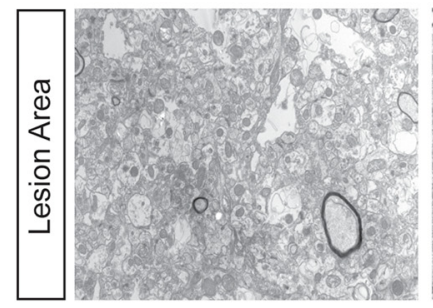

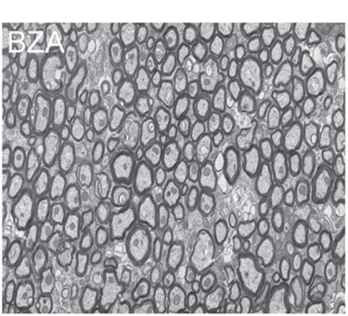

\section{f}
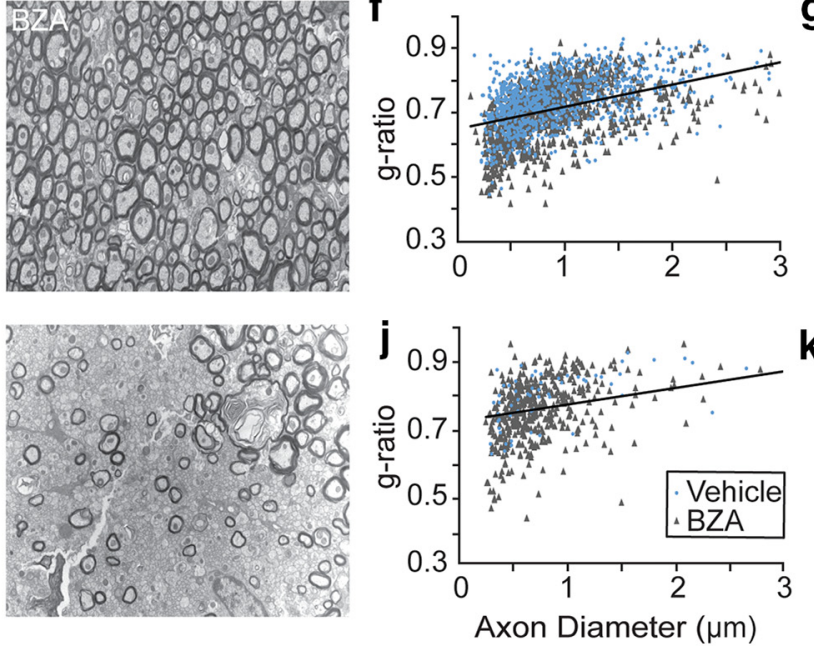
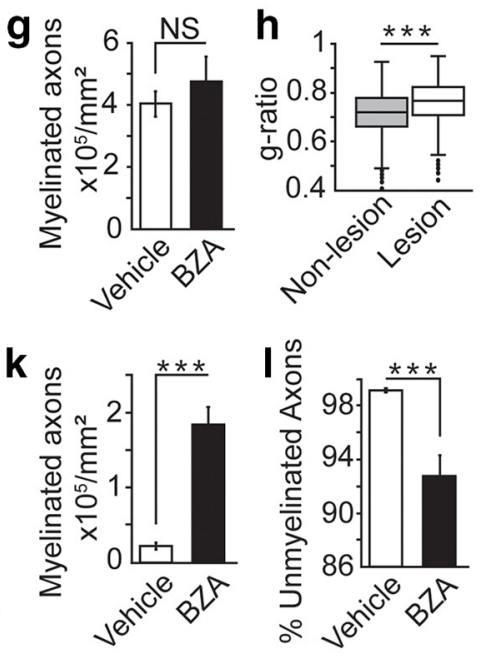

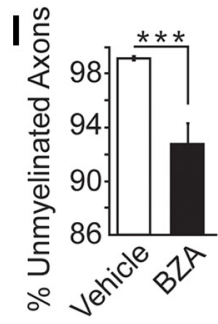

Figure 3. BZA enhances and accelerates remyelination in an in vivo focal demyelination model $a$, Schematic showing location of lysolecithin injection into the corpus callosum at the position: AP: $-1.04, \mathrm{ML}: 1$, and DV: -1.75 from bregma. $\boldsymbol{b}$, Representative images of lesions within the corpus callosum of adult WT mice. Lesions were identified by the density of cell nuclei (DAPI) and included based on NF staining for intact axons and their boundaries are demarcated in white. Animals were given either vehicle or BZA at $10 \mathrm{mg} / \mathrm{kg} / \mathrm{d}$ for $7 \mathrm{~d}$ beginning at $1 \mathrm{dpl}$ and perfused at $10 \mathrm{dpl}$. They

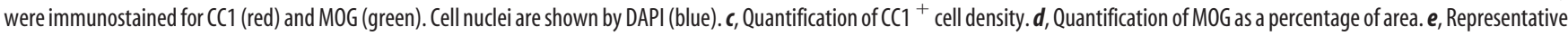
images of myelinated axon density in nonlesioned areas in vehicle- and BZA-treated mice. $\boldsymbol{f}$, Scatterplot of axon diameter plotted against corresponding g-ratio of vehicle- and BZA-treated mice demonstrating no difference in g-ratio between groups. $\boldsymbol{g}$, Quantification of myelinated axon density within the nonlesioned area. $\boldsymbol{h}$, Quantification of average g-ratio within lesioned and nonlesioned area. $\boldsymbol{i}$, Representative images of remyelinated axon density within the lesioned area in vehicle- and BZA-treated mice. $\boldsymbol{j}$, Scatterplot of axon diameter plotted against corresponding g-ratio of vehicle- and BZA-treated mice in the lesioned area. $\boldsymbol{k}$, Quantification of remyelinated axon density within lesioned areas in vehicle- and BZA-treated mice. I, Quantification of the percentage of unmyelinated axons in lesion areas in vehicle- and BZA-treated mice. Error bars indicate SEM. ${ }^{* *} p<0.01$, ${ }^{* * *} p<0.001$. For immunostaining experiments, $n=5$ animals per group and $n=10$ sections analyzed per animal; for EM analyses, $n=2$ animals per group and $n=6$ images analyzed per animal.

drocytes and myelin)-expressing oligodendrocytes $(p=1.12 \times$ $10^{-16}$, paired $t$ test) (Fig. $2 b$ ). Moving from the original screen using rat OPCs to human OPCs allowed us not only to validate BZA's potent actions on differentiation, but also ensured that the effect is robust in human cells.

To gain a greater understanding of BZA's mechanism of action, we first determined a dose-response curve by treating purified OPCs isolated from postnatal rat pups with a range of BZA concentrations for $48 \mathrm{~h}$ (Fig. $2 c$ ). We saw significant enhancement of OPC differentiation beginning at $5 \mathrm{nM}(p=0.013$, oneway ANOVA with Tukey's multiple-comparisons test) with increasing effects up to $500 \mathrm{nM}\left(10 \mathrm{nM} p=7.77 \times 10^{-8} ; 20 \mathrm{nM}\right.$ $p=5.70 \times 10^{-14} ; 50 \mathrm{~nm} p=4.70 \times 10^{-14} ; 500 \mathrm{nм} p=8.00 \times$
$10^{-15}$, one-way ANOVA with Tukey's multiple-comparisons test) (Fig. 2d). This dose-response curve within the single nanomolar range is promising for the future $\mathrm{EC} / \mathrm{IC}_{50}$ when moving forward to human dosage predictions. A subset of others SERMs showed this effect, whereas others-and estrogens- did not, illuminating the need for target validation (estriol $p=0.999$; arzoxifene $p=0.991$; diarylproprionitrile $p=0.946$; lasofoxifene $p=1.81 \times 10^{-6}$; tamoxifen $p=1.55 \times 10^{-11}$; bazedoxifene $p=$ $1.48 \times 10^{-13}$, one-way ANOVA with Tukey's multiplecomparisons test) (Figs. 1, 2e). Following the promising results of BZA treatment on isolated OPCs, we assessed whether the resultant oligodendrocytes were functional. To this end, we used a coculture system consisting of DRGs, which serve as axonal sub- 

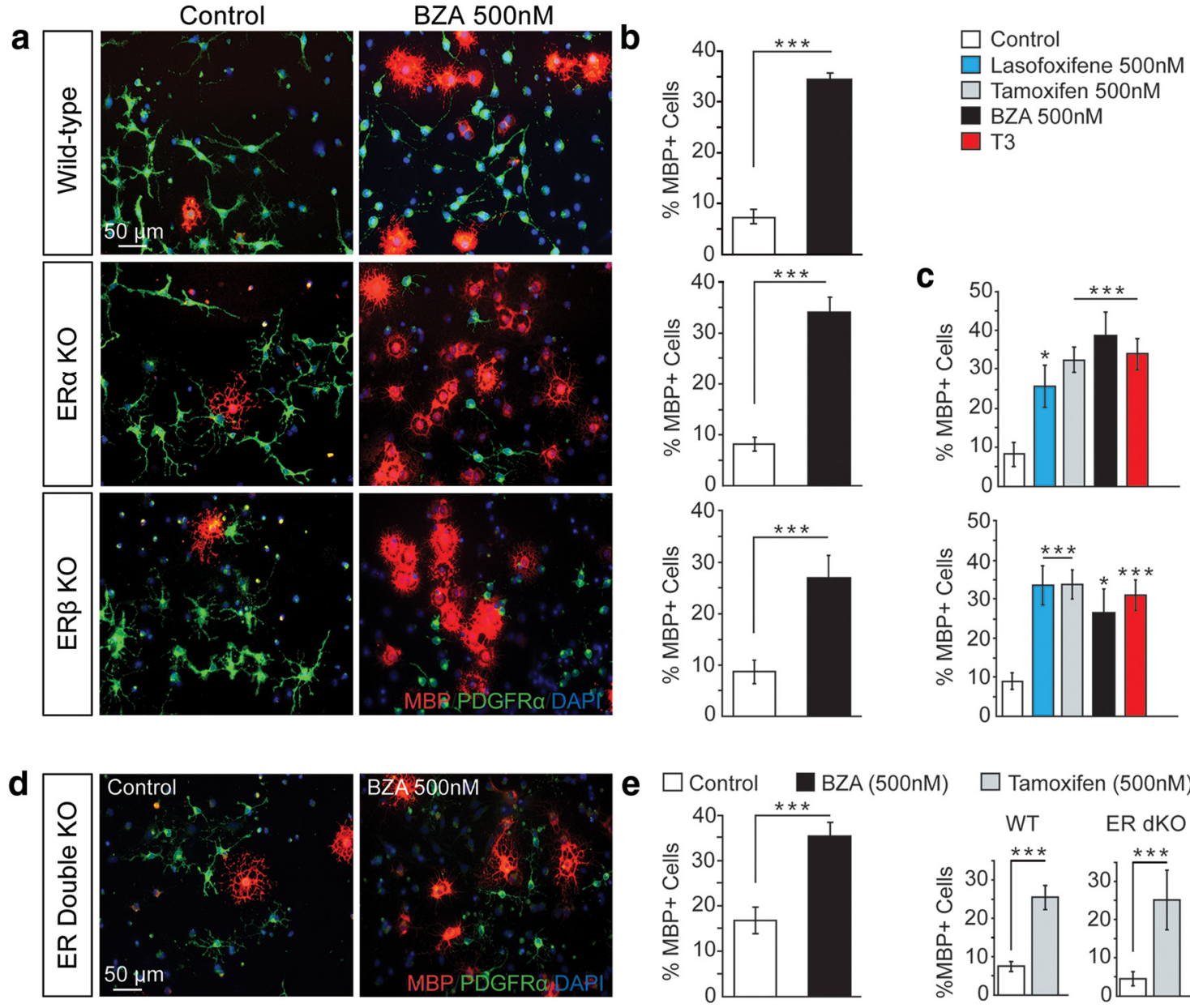

Figure 4. BZA enhances OPC differentiation independently of the ER $\boldsymbol{a}$, Representative images of control and BZA-treated ( $500 \mathrm{~nm}) \mathrm{WT}$, ER $\alpha$, and ER $\beta$ KO OPCs, treated for 2 d and immunostained for MBP (red) and PDGFR $\alpha$ (green). Cell nuclei are shown by DAPI (blue). $\boldsymbol{b}$, Quantification of $\mathrm{MBP}^{+}$cells over total cells in WT, ER $\alpha$, and ER $\beta$ KO OPC cultures treated with control or BZA (500 nM). c, Quantification of $\mathrm{MBP}^{+}$cells over total cells in ER $\alpha$ and ER $\beta$ KO OPC cultures treated with various SERMs ( $\left.500 \mathrm{~nm}\right)$, as well as the positive control T3 $(1 \times)$. $\boldsymbol{d}$, Representative images of control and BZA-treated ( $500 \mathrm{nm)} \mathrm{OPCs} \mathrm{from} \mathrm{ER} \alpha$ and ER $\beta$ DKO animals treated for $2 \mathrm{~d}$ and immunostained for MBP (red) and PDGFR $\alpha$ (green). Cell nuclei are shown by DAPI (blue). $e$, Quantification of MBP ${ }^{+}$ cells over total cells (DAPI ${ }^{+}$nuclei) in ER-DKO OPC cultures of BZA- and tamoxifen $(500 \mathrm{~nm})$-treated groups. For all quantifications, error bars indicate SEM. ${ }^{*} p<0.05,{ }^{* * *} p<0.001$. For all experiments, $n=3$ temporally separate trials and $n>3$ coverslips per group per trial.

strates for myelination (Chan et al., 2004), and OPCs (Fig. 2f). BZA-treated cocultures had significantly more CC1 (a marker of differentiated oligodendrocytes) and MBP-colabeled cells that myelinate DRG axons ( $p=3.00 \times 10^{-7}$, paired $t$ test) (Fig. $2 g$ ). In addition, there were significantly more myelin internodes in the BZA-treated cocultures $\left(p=4.98 \times 10^{-9}\right.$, paired $t$ test $)$ (Fig. $2 i)$. Therefore, BZA enhanced not only OPC differentiation at nanomolar ranges, but also myelination of axonal substrates in culture.

We next assessed treatment in a demyelinating disease model. Our focus on BZA was twofold: we wanted to validate its cellautonomous effects on OPCs and ensure its translational potential in demyelinating disease. To this end, we made use of the lysolecithin focal demyelination model. This model shows minimal immune cell involvement and lesions will fully remyelinate following a well established timeline for repair (Fancy et al., 2011). We induced a focal demyelinating lesion in the corpus callosum of WT adult mice (Fig. $3 a$ ) and assessed remyelination at $10 \mathrm{dpl}$ induction based on the density of CC1-expressing oligodendrocytes within the lesion area and percentage of myelinated area as determined by MOG (a marker of mature oligodendrocytes and myelin) within the lesion (Fig. 3b). Mice were treated with BZA or vehicle from 1 to $7 \mathrm{dpl}$. We saw a significantly enhanced number of CC1-expressing cells ( $p=$ 0.002 , paired $t$ test) (Fig. $3 c$ ) and a significantly greater myelinated area ( $p=0.007$, paired $t$ test) within the lesion (Fig. $3 d)$ in the BZA-treated group, indicating that BZA significantly enhanced OPC differentiation and accelerated repair in this focal demyelination model. We further analyzed the effects of BZA on remyelination at $10 \mathrm{dpl}$ using $\mathrm{EM}$ of lysolecithin-treated animals. It is important to note that the typical time point for remyelination by electron microscopy is $14 \mathrm{dpl}$. However, we chose to examine remyelination at $10 \mathrm{dpl}$ because we were trying to identify an exceptionally potent effect on remyelination. Normally at this point, there is almost no remyelination in vehicle controls. Mice were treated with BZA from $1 \mathrm{dpl}$ through $7 \mathrm{dpl}$. In the nonlesioned area, we saw no differences in myelination density nor g-ratios ( $p=0.445$, paired $t$ test), indicating that BZA does not result in abnormal myelination (Fig. $3 e-g$ ). Remyelinated axons are indicated by a higher g-ratio, representing thinner myelin. We noted significantly higher g-ratios in the lesioned areas compared with the nonlesioned areas $\left(p=1.09 \times 10^{-25}\right.$, paired $t$ test), indicating that remyelination had occurred within the lesions (Fig. 3h). In the lesioned area, we saw a significant difference in the number of remyelinated axons within the lesion site in BZA-treated mice compared with vehicle-treated mice $(p=$ 

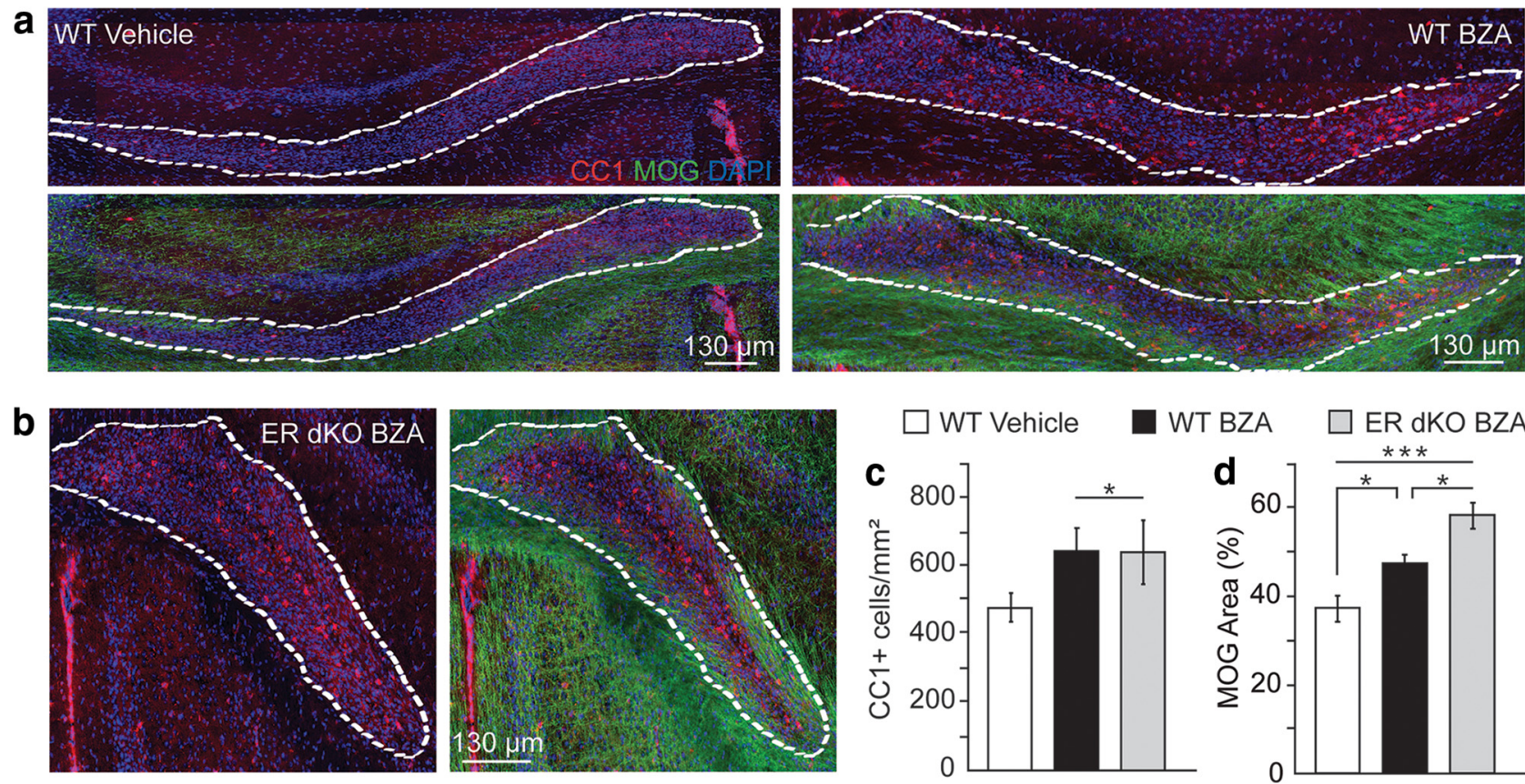

Figure 5. BZA accelerates remyelination independently of the ER $\boldsymbol{a}$, Representative images of identified lesions within the corpus callosum of WT mice. $\boldsymbol{b}$, Representative images of identified lesions within the corpus callosum of ER-DKO mice. Lesions were identified by the density of cell nuclei and their boundaries demarcated in white. Animals were given either vehicle or BZA at 10 $\mathrm{mg} / \mathrm{kg} / \mathrm{d}$ for $7 \mathrm{~d}$ following lesion induction and killed and perfused at $10 \mathrm{dpl}$. They were immunostained for $\mathrm{CC} 1$ (red) and MOG (green). Cell nuclei are shown by DAPI (blue). $c$, Quantification of $C \mathrm{C} 1^{+}$ cell density within the lesion area of WT and ER-DKO animals. $\boldsymbol{d}$, Quantification of MOG as a percentage of area in WT and ER-DKO animals. For all quantifications, error bars indicate SEM. ${ }^{*} p<0.05$, ${ }^{* * *} p<0.001$. For all experiments, $n>4$ animals per group and $n=10$ sections analyzed per animal.

$3.05 \times 10^{-6}$, paired $t$ test) (Fig. $3 i-k$ ). This was accompanied by a concomitant significant decrease in the percentage of unmyelinated axons $\left(p=4.00 \times 10^{-4}\right.$, paired $t$ test) (Fig. $\left.3 l\right)$. This indicates a significant enhancement and acceleration of remyelination in BZA-treated animals.

SERMs have been developed to differentially agonize or antagonize nuclear $\mathrm{ER} \alpha$ and $\mathrm{ER} \beta$ in selected tissues in response to the more pleiotropic and potentially harmful effects of estrogen treatment (Rossouw et al., 2002; Chlebowski et al., 2003). Previous studies have relied heavily on an assumption of SERMs' specificity and reported a reliance on the ERs to mediate effects on remyelination and neuroprotection (Crawford et al., 2010; Khalaj et al., 2013; Moore et al., 2014). However, the authenticity of many antibodies used to determine ER presence has recently been challenged (Andersson et al., 2017), highlighting the need for mechanism validation in $\mathrm{KO}$ models with appropriate outcome measures. Further, our results demonstrate that certain SERMs promote differentiation, whereas others, as well as estrogens, do not (Figs. 1, 2e). Together, these findings bring into question the specific receptor target and raise the possibility for off-target effects. We therefore sought to determine the mechanism of action for BZA. BZA acts as an agonist and antagonist on both nuclear ERs (McKeand et al., 2014). Therefore, we isolated OPCs from total ER $\beta$ and $\mathrm{ER} \alpha \mathrm{KO}$ mice and assessed OPC differentiation after treatment with BZA for $48 \mathrm{~h}$ (Fig. $4 a$ ). We were surprised to see significantly enhanced OPC differentiation across all treatment groups to the same magnitude as WT cells $\left(\mathrm{ER} \alpha p=1.25 \times 10^{-10} ; \mathrm{ER} \beta p=4.00 \times 10^{-4}\right.$, paired $t$ test $)$ (Fig. $4 b)$, indicating that BZA does not induce OPC differentiation through ER $\alpha$ or ER $\beta$. This effect persisted for all SERMs found to have positive effects on differentiation in WT OPCs (ER $\alpha$ : lasofoxifene $p=0.011$; tamoxifen $p=3.37 \times 10^{-6}$; bazedoxifene $p=$ $2.62 \times 10^{-10} ; \mathrm{ER} \beta$ : lasofoxifene $p=2.80 \times 10^{-5}$; tamoxifen $p=$
$2.26 \times 10^{-4}$; bazedoxifene $p=0.029$, one-way ANOVA with Tukey's multiple-comparisons test), as well as the positive control T3 $\left(\mathrm{ER} \alpha: p=4.52 \times 10^{-5} ; \mathrm{ER} \beta p=1.47 \times 10^{-3}\right.$, one-way ANOVA with Tukey's multiple-comparisons test) (Fig. $4 c$ ). To ensure there was no compensation from the complementary receptor, we generated $\mathrm{ER} \alpha$ and $\mathrm{ER} \beta$ double-KO animals (ERDKO) (Fig. 4d). After treatment with BZA or tamoxifen, we again saw significant enhancement of OPC differentiation (bazedoxifene $p=3.00 \times 10^{-5}$; tamoxifen $p=7.67 \times 10^{-7}$, paired $t$ test) (Fig. 4e). This led us to conclude that BZA and other SERMs exert potent effects on OPC differentiation independently of their intended nuclear ER targets.

To reinforce this finding, specifically in light of the lack of $\mathrm{KO}$ validations in the past, we repeated the lysolecithin experiments in ER-DKO mice (Fig. 5b) alongside WT controls (Fig. 5a). Here again, BZA enhanced the number of $\mathrm{CC}^{+}{ }^{+}$cells within the lesion (WT BZA $p=0.032$; ER-DKO BZA $p=0.028$, one-way ANOVA with Tukey's multiple-comparisons test) (Fig. $5 c$ ), as well as the percentage of myelinated area (WT BZA $p=0.015$; ER-DKO BZA $p=$ $8.27 \times 10^{-6}$; WT BZA vs ER-DKO BZA $p=0.015$, one-way ANOVA with Tukey's multiple-comparisons test) (Fig. $5 d$ ), thereby still accelerating remyelination in the ER-DKO animals to the same degree as WT animals. Together, these findings clearly indicate that BZA does not require either nuclear ER to mediate its effects on oligodendrocyte differentiation and remyelination.

In light of the surprising lack of requirement for either ER for BZA to mediate its effect on OPC differentiation, we took steps to gain greater clarity into the mechanism of action and to isolate SERMs' veritable target. Due to the great difficulty of identifying targets and inherent promiscuity of small molecules, we moved forward with a bioinformatics approach to determine candidate target potentials. Using a previously published paradigm that accurately predicts interactions with $>97 \%$ efficiency (Wang et 


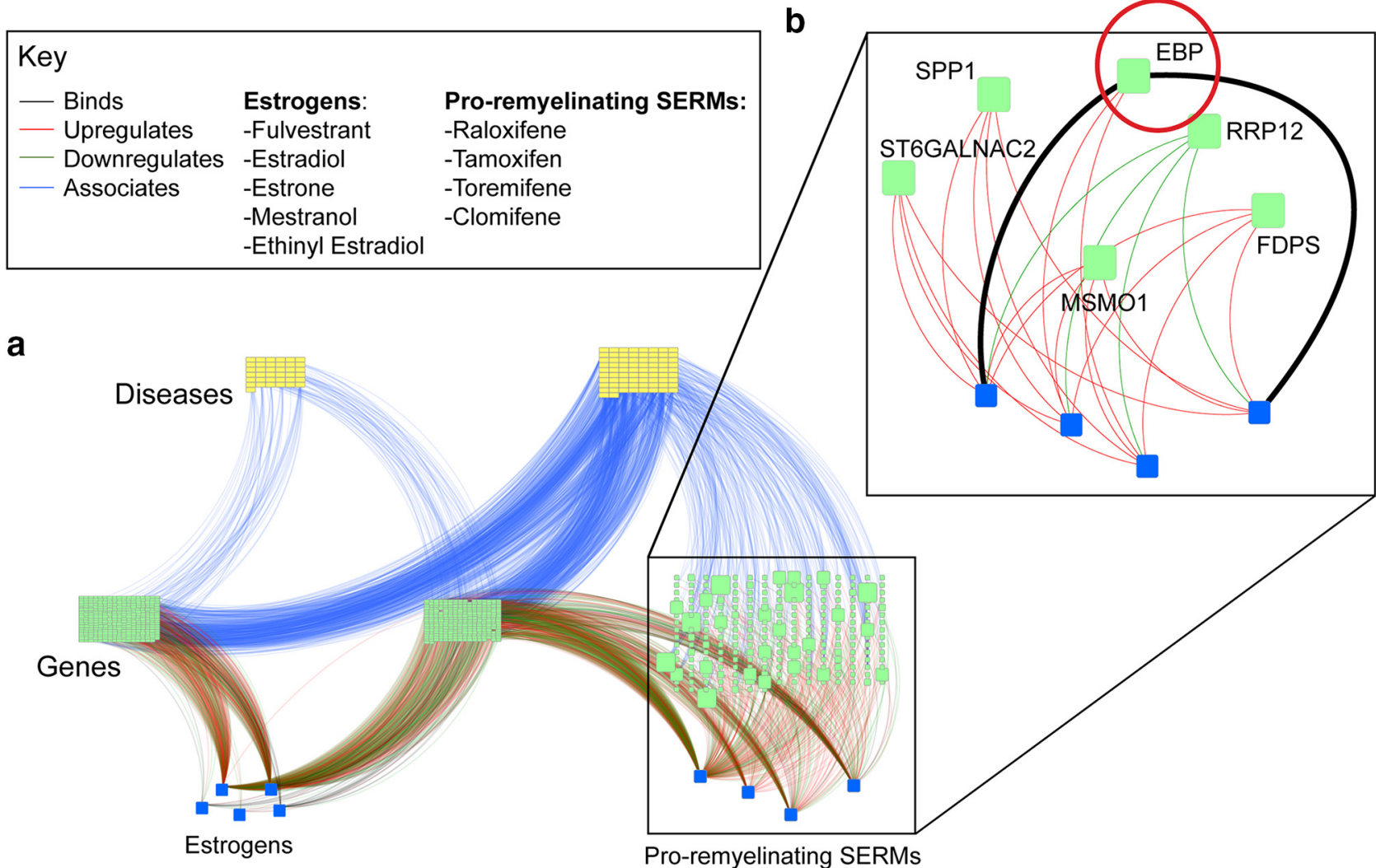

C
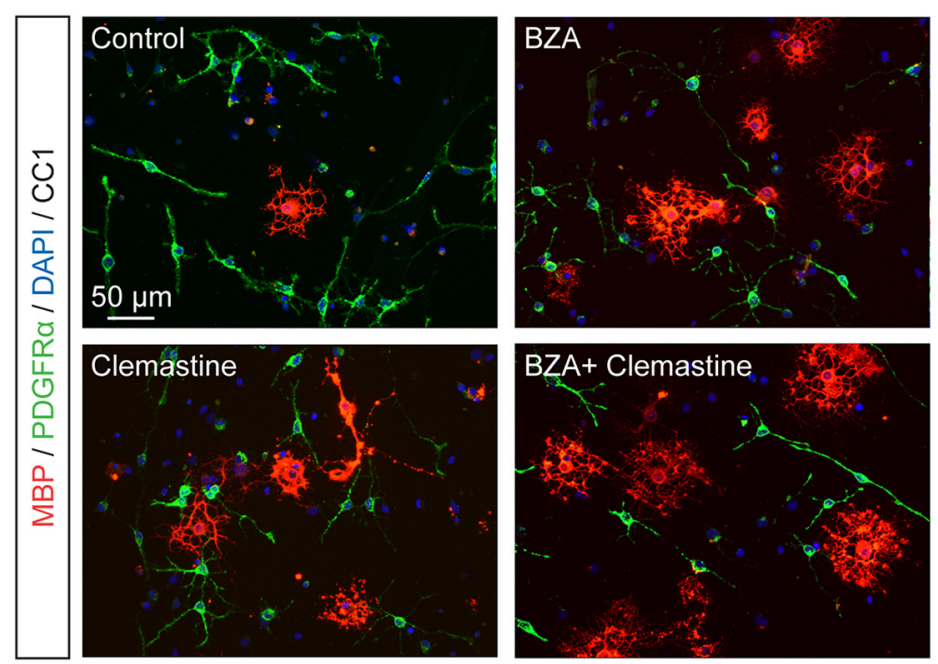

d
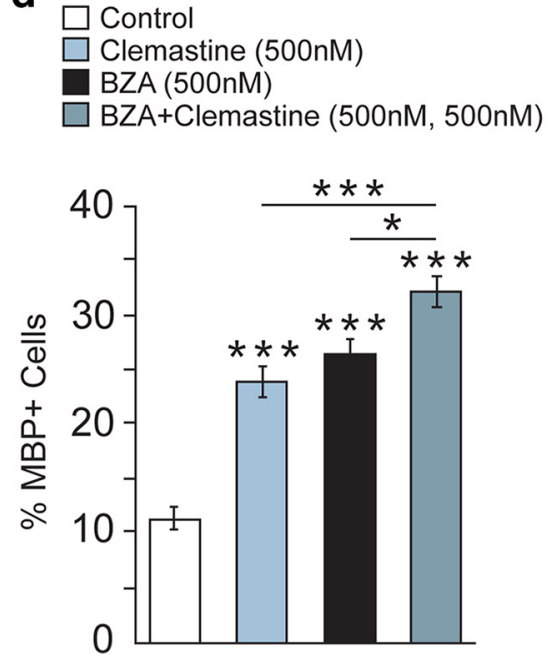

Figure 6. Bioinformatics profiling implicates set of candidate targets positing unique pathway for SERMs $\boldsymbol{a}$, Network of first-degree (genes/proteins; green squares) and second-degree (diseases; yellow squares) neighbors of select FDA-approved pro-remyelinating and non-remyelinating compounds (blue squares) was generated using HetioNet (Himmelstein et al., 2017) and visualized using $\mathrm{iCTNET}$ (Wang et al., 2015). $\boldsymbol{b}$, Ggenes with the highest number of connections to the four pro-remyelinating compounds are labeled and the resulting subnetwork is enlarged in the inset. EBP is the predicted binding partner of pro-remyelinating compounds (raloxifene and tamoxifen) based on recently published data. ST6GALNAC2, SPP1, MSM0, and FDPS are upregulated and RRP12 is downregulated by all four compounds. Binding data were retrieved from BindingDB, DrugBank, and DrugCentral. Only binding relationships to single proteins with affinities of at least $1 \mu \mathrm{M}$ (as determined by $K_{\mathrm{d}}, K_{\mathrm{i}}$, or $\mathrm{C}_{50}$ ) were selected from the 0 ctober 2015 release of BindingDB. Target, carrier, transporter, and enzyme interactions with single proteins (i.e., excluding protein groups) were extracted from DrugBank 4.2. Regulatory effects of compounds on genes was based on the LINCS L1000 dataset and computed as in Himmelstein et al. (2017). Key terms: "Binding" indicates physical evidence that compound binds protein used to map drugs onto proteins; "association" indicates statistical evidence that gene confers risk to the disease and is used to map genes onto diseases. $\boldsymbol{C}$, Representative images of control, BZA-treated (500 nM), clemastine-treated ( $500 \mathrm{~nm}$ ), and BZA + clemastine-treated ( $500 \mathrm{~nm}, 500 \mathrm{~nm})$ OPCs treated for $2 \mathrm{~d}$ and immunostained for MBP (red) and PDGFR $\alpha$ (green). Cell nuclei are shown by DAPI (blue). $\boldsymbol{d}$, Quantification of $\mathrm{MBP}^{+}$cells over total cells (DAPI ${ }^{+}$nuclei) in OPC cultures of BZA-treated, clemastine-treated, and BZA + clemastinetreated ( $500 \mathrm{~nm}$ ) groups. For all quantifications, error bars indicate SEM. ${ }^{*} p<0.05,{ }^{* * *} p<0.001$. For all experiments, $n=4$ temporally separate trials and $n>3$ coverslips per group per trial. 
al., 2015; Himmelstein et al., 2017), integrating 29 databases and 1500 compounds mapped into a heterogeneous network (Chen et al., 2001; Law et al., 2014; Gilson et al., 2016; Ursu et al., 2017), we selectively clustered FDA-approved SERMs with positive effects on OPC differentiation, SERMs with negative effects, and estrogens. Based on predicted connections, these clusters mapped onto distinct sets of potential molecular targets (Fig. 6a). Six unique candidate targets emerged with predicted interactions to all four candidate FDA-approved SERMs (Fig. 6b). Of these possible targets, EBP was of particular interest.

EBP (encoding $3 \beta$-hydroxysteroid- $\triangle 8$ - $\triangle 7$-sterol isomerase) is an important enzyme in the biosynthesis of cholesterol. It catalyzes the isomerization of zymosterol (cholesta-8(9),24dien-3 $\beta$-ol) to lathosterol (cholesta-7,24-dien-3 $\beta$-ol) and its inhibition results in the accumulation of the 8,9-unsaturated sterol zymosterol. This inhibition and subsequent accumulation of an 8,9-unsaturated sterol has been recently implicated as one of three candidate steps within the cholesterol biosynthesis pathway (acting through a redundant mechanism) that would result in enhanced OPC differentiation (Hubler et al., 2018). Tamoxifen has previously been shown to inhibit EBP (Gylling et al., 1995; Moebius et al., 1998; Korade et al., 2016). More importantly, the inhibition of any of these three candidate enzymes, resulting in accumulation of an 8,9-unsaturated sterol, has been implicated as a common target for all previously publicly identified proremyelinating compounds (Hubler et al., 2018). Through this novel, unbiased approach of bioinformatics profiling, we independently identified a candidate target that has only recently been implicated in remyelination. These converging independent reports bolster the impetus to pursue this likely target in OPC differentiation and remyelination.

To provide further insight into the divergent mechanism of $\mathrm{BZA}$, we investigated whether BZA had an additive effect on OPC differentiation in combination with a previously validated proremyelinating compound known to operate through a distinct pathway. Here, we demonstrate that, in combination with clemastine (at maximal dose), a compound previously shown to promote OPC differentiation by antagonizing the muscarinic acetylcholine receptor 1 subtype (Mei et al., 2016), BZA (at maximal dose) exerts an additive effect on OPC differentiation greater than either compound on their own (clemastine vs control: $2.24 \times 10^{-9}$; BZA vs control: $5.90 \times 10^{-13}$; BZA + clemastine vs control: $4.40 \times 10^{-14}$; BZA + clemastine vs clemastine: $1.19 \times 10^{-4} ; \mathrm{BZA}+$ clemastine vs BZA: 0.01 , one-way ANOVA with Tukey's multiple-comparisons test) (Fig. $6 c, d$ ), thus indicating that these two compounds mediate their effects on OPC differentiation through different targets.

\section{Discussion}

Remyelination represents a significant obstacle within the MS population. This paramount failure of repair sets forward the path to axonal loss and thereby disability progression (Irvine and Blakemore, 2008; Trapp and Nave, 2008). Given the lack of therapies to promote repair, our results from a clinically available drug, which is already readily given to patients for the alleviation of vasomotor menopausal symptoms, present an exciting opportunity to intervene. BZA is currently FDA-approved in combination with conjugated estrogens as the tissue-selective estrogen complex (TSEC) Duavee and is EMA-approved on its own (Wyeth Europa, Ltd., Conbriza [package insert], 2009). Of paramount importance, BZA has proven very tolerable in clinical trials, setting it apart from other SERMs (Mirkin and Komm, 2013; McKeand et al., 2014). In fact, the prominent side effects of this drug would be positive and reflect its current indication: osteoporosis prevention and alleviation of potential vasomotor menopausal symptoms (Wyeth Pharmaceuticals, Inc., and Pfizer, Inc.: Duavee [package insert], 2013; McKeand et al., 2014). We are currently well under way for a phase II clinical trial.

Although BZA is currently only approved for use in women, women represent $75 \%$ of the population who develop MS. However, unexpectedly and fortuitously, we found that BZA and other SERMs do not act through the ERs to promote OPC differentiation and remyelination, as would be expected given current dogma in the field. The field of hormonal research into CNS diseases has dedicated countless money and energy into estrogens as treatment for MS symptoms, with only conflicting and underwhelming results. Our study provides incontrovertible evidence that the ER as a target is not a lucrative avenue of pursuit for remyelinating therapies. The off-target receptor through which BZA exerts its effects within the single nanomolar range presents exciting opportunities for future drug development, which would substantially expand the population of patients beyond what has been previously proposed. Although as yet unverified, this study provides complementary results for a recently published study positing three candidate enzymes as possible targets for all previously publicly identified pro-remyelinating therapies (Hubler et al., 2018). Our novel approach to identifying these compounds uses a predictive, machine-learning algorithm and does not provide definitive proof, but indicates likely possibilities worthy of future pursuit. These findings present exciting opportunities for the field to not only advance current drugs such as SERMs to human trials, but also to understand the importance of the cholesterol biosynthesis pathway in OPC differentiation for the development of drugs with greater and targeted affinity for this pathway.

\section{References}

Andersson S, Sundberg M, Pristovsek N, Ibrahim A, Jonsson P, Katona B, Clausson CM, Zieba A, Ramström M, Söderberg O, Williams C, Asplund A (2017) Insufficient antibody validation challenges oestrogen receptor beta research. Nat Commun 8:15840.

Chan JR, Watkins TA, Cosgaya JM, Zhang C, Chen L, Reichardt LF, Shooter EM, Barres BA (2004) NGF controls axonal receptivity to myelination by schwann cells or oligodendrocytes. Neuron 43:183-191.

Chen X, Liu M, Gilson MK (2001) BindingDB: A Web-Accessible Molecular Recognition Database. Combinatorial chemistry and high throughput screening. 4:719-725.

Chlebowski RT, Hendrix SL, Langer RD, Stefanick ML, Gass M, Lane D, Rodabough RJ, Gilligan MA, Cyr MG, Thomson CA, Khandekar J, Petrovitch H, McTiernan A, McTiernan A (2003) Influence of estrogen plus progestin on breast cancer and mammography in healthy postmenopausal women: the Women's health initiative randomized trial. JAMA 289:3243-3253

Crawford AH, Tripathi RB, Foerster S, McKenzie I, Kougioumtzidou E, Grist M, Richardson WD, Franklin RJ (2016) Pre-existing mature oligodendrocytes do not contribute to remyelination following toxin-induced spinal cord demyelination. Am J Pathol 186:511-516.

Crawford DK, Mangiardi M, Song B, Patel R, Du S, Sofroniew MV, Voskuhl RR, Tiwari-Woodruff SK (2010) Oestrogen receptor $\beta$ ligand: a novel treatment to enhance endogenous functional remyelination. Brain 133: 2999-3016.

Douvaras P, Fossati V (2015) Generation and isolation of oligodendrocyte progenitor cells from human pluripotent stem cells. Nat Protoc 10:11431154.

Fancy SP, Harrington EP, Yuen TJ, Silbereis JC, Zhao C, Baranzini SE, Bruce CC, Otero JJ, Huang EJ, Nusse R, Franklin RJ, Rowitch DH (2011) Axin2 as regulatory and therapeutic target in newborn brain injury and remyelination. Nat Neurosci 14:1009-1016.

Frischer JM, Weigand SD, Guo Y, Kale N, Parisi JE, Pirko I, Mandrekar J, Bramow S, Metz I, Brück W, Lassmann H, Lucchinetti CF (2015) Clin- 
ical and pathological insights into the dynamic nature of the white matter multiple sclerosis plaque. Ann Neurol 78:710-721.

Gilson MK, Liu T, Baitaluk M, Nicola G, Hwang L, Chong J (2016) BindingDB in 2015: A public database for medicinal chemistry computational chemitry and systems pharmacology. Nucleic Acid Res 44:D1045-D1053.

Gonzalez GA, Hofer MP, Syed YA, Amaral AI, Rundle J, Rahman S, Zhao C, Kotter MR (2016) Tamoxifen accelerates repair of demyelinated lesions in the central nervous system. Sci Rep 6:31599.

Gylling H, Pyrhönen S, Mäntylä E, Mäenpää H, Kangas L, Miettinen TA (1995) Tamoxifen and toremifene lower serum cholesterol by inhibition of delta 8-cholesterol conversion to lathosterol in women with breast cancer. J Clin Oncol 13:2900-2905.

Hauser SL, Chan JR, Oksenberg JR (2013) Multiple sclerosis: prospects and promise. Ann Neurol 74:317-327.

Himmelstein DS (2016) Dhimmel/Doaf V1.0: Processing The Doaf Database Of Gene-Disease Associations. Available at https://zenodo.org/ record/48427\#.W7VH1xNKiCU.

Himmelstein DS, Lizee A, Hessler C, Brueggeman L, Chen SL, Hadley D, Green A, Khankhanian P, Baranzini SE (2017) Systematic integration of biomedical knowledge prioritizes drugs for repurposing. eLife 6:e26726.

Hubler Z, Allimuthu D, Bederman I, Elitt MS, Madhavan M, Allan KC, Shick HE, Garrison E, T Karl M, Factor DC, Nevin ZS, Sax JL, Thompson MA, Fedorov Y, Jin J, Wilson WK, Giera M, Bracher F, Miller RH, Tesar PJ, et al. (2018) Accumulation of 8,9-unsaturated sterols drives oligodendrocyte formation and remyelination. Nature 560:372-376.

Irvine KA, Blakemore WF (2008) Remyelination protects axons from demyelination-associated axon degeneration. Brain 131:1464-1477.

Khalaj AJ, Yoon J, Nakai J, Winchester Z, Moore SM, Yoo T, Martinez-Torres L, Kumar S, Itoh N, Tiwari-Woodruff SK (2013) Estrogen receptor (ER) $\beta$ expression is required for attenuation of clinical disease by an ER $\beta$ ligand. Proc Natl Acad Sci U S A 110:19125-19130.

Korade Z, Kim HY, Tallman KA, Liu W, Koczok K, Balogh I, Xu L, Mirnics K, Porter NA (2016) The effect of small molecules on sterol homeostasis: measuring 7-dehydrocholesterol in DHCR7-deficient Neuro2a cells and human fibroblasts. J Med Chem 59:1102-1115.

Kuhlmann T, Miron V, Cui Q, Cuo Q, Wegner C, Antel J, Brück W (2008) Differentiation block of oligodendroglial progenitor cells as a cause for remyelination failure in chronic multiple sclerosis. Brain 131:1749-1758.

Law V, Knox C, Djoumbou Y, Jewison T, Guo AC, Liu Y, Maciejewksi A, Arndt D, Wilson M, Neveu V, Tang A, Gabriel G, Ly C, Adamjee S, Dame ZT, Han B, Zhou Y, Wishart DS (2014) DrugBank 4.0: shedding new light on drug metabolism. Nucleic Acid Res 42:D1091-D1097.

MacArthur J, Bowler E, Cerezo M, Gil L, Hall P, Hastings E, Junkins H, McMahon A, Milano A, Morales J, Pendlington ZM, Welter D, Burdett T, Hindorff L, Flicek P, Cunningham F, Parkinson H (2017) The new NHGRI-EBI Catalog of published genome-wide association studies (GWAS Catalog). Nucleic Acids Res 45:D896-D901.

McKeand WE, Orczyk GP, Ermer JC, Chines AA (2014) A double-blind, randomized, ascending, multiple-dose study of bazedoxifene in healthy postmenopausal women. Clin Pharmacol Drug Dev 3:262-269.

Mei F, Fancy SPJ, Shen YA, Niu J, Zhao C, Presley B, Miao E, Lee S, Mayoral SR, Redmond SA, Etxeberria A, Xiao L, Franklin RJM, Green A, Hauser
SL, Chan JR (2014) Micropillar arrays as a high-throughput screening platform for therapeutics in multiple sclerosis. Nat Med 20:954-960.

Mei F, Lehmann-Horn K, Shen YA, Rankin KA, Stebbins KJ, Lorrain DS, Pekarek K, A Sagan S, Xiao L, Teuscher C, von Büdingen HC, Wess J, Lawrence JJ, Green AJ, Fancy SP, Zamvil SS, Chan JR (2016) Accelerated remyelination during inflammatory demyelination prevents axonal loss and improves functional recovery. eLife 5:e18246.

Mirkin S, Komm BS (2013) Tissue-selective estrogen complexes for postmenopausal women. Maturitas 76:213-220.

Moebius FF, Reiter RJ, Bermoser K, Glossmann H, Cho SY, Paik YK (1998) Pharmacological analysis of sterol delta8-delta7 isomerase proteins with [3H] ifenprodil. Mol Pharmacol 54:591-598.

Moore SM, Khalaj AJ, Kumar S, Winchester Z, Yoon J, Yoo T, MartinezTorres L, Yasui N, Katzenellenbogen JA, Tiwari-Woodruff SK (2014) Multiple functional therapeutic effects of the estrogen receptor $\beta$ agonist indazole-chloride in a mouse model of multiple sclerosis. Proc Natl Acad Sci U S A 111:18061-18066.

Nair AB, Jacob S (2016) A simple practice guide for dose conversion between animals and human. J Basic Clin Pharm 7:27-31.

National Institutes of Health (2018) LINCS100 Project. Available at http://www.lincsproject.org/LINCS/about.

Perez EA (2007) Safety profiles of tamoxifen and the aromatase inhibitors in adjuvant therapy of hormone-responsive early breast cancer. Ann Oncol 18:viii26-viii35.

Pickar JH, Yeh IT, Bachmann G, Speroff L (2009) Endometrial effects of a tissue selective estrogen complex containing bazedoxifene/conjugated estrogens as a menopausal therapy. Fertil Steril 92:1018-1024.

Piñero J, Bravo À, Queralt-Rosinach N, Gutiérrez-Sacristán A, Deu-Pons J, Centeno E, García-García J, Sanz F, Furlong LI (2017) DisGeNET: a comprehensive platform integrating information on human diseaseassociated genes and variants. Nucleic Acids Res 45:D833-D839.

Pletscher-Frankild S, Pallejà A, Tsafou K, Binder JX, Jensen LJ (2015) DISEASES: text mining and data integration of disease-gene associations. Methods 74:83-89.

Rossouw JE, Anderson GL, Prentice RL, LaCroix AZ, Kooperberg C, Stefanick ML, Jackson RD, Beresford SA, Howard BV, Johnson KC, Kotchen JM, Ockene J, Ockene J (2002) Risks and benefits of estrogen plus progestin in healthy postmenopausal women: principal results from the Women's health initiative randomized controlled trial. JAMA 288:321-333.

Trapp BD, Nave KA (2008) Multiple sclerosis: an immune or neurodegenerative disorder? Annu Rev Neurosci 31:247-269.

Ursu O, Holmes J, Knockel J, Bologa CG, Yang JJ, Mathias SL, Nelson SJ, Oprea TI (2017) DrugCentral: online drug compendium. Nucleic Acid Res 45:D932-D939.

Wallin M (2017) The prevalence of multiple sclerosis in the United States: a population-based healthcare database approach. ECTRIMS abstract. Available at https://onlinelibrary.ectrims-congress.eu/ectrims/2017/ ACTRIMS-ECTRIMS2017/199999/mitchell.t.wallin.the.prevalence.of. multiple.sclerosis.in.the.united.states.a.html.

Wang L, Himmelstein DS, Santaniello A, Parvin M, Baranzini SE (2015) iCTNet2: integrating heterogeneous biological interactions to understand complex traits. F1000Res 4:485. 